
\title{
PAINEL DE ESPECIALISTAS Linha de cuidados no tratamento da
EXPERT PANEL \\ asma: informações úteis para o gestor
}

\author{
Comprehensive care in asthma treatment: useful \\ information for the health-care manager
}

Marcos Santos 1,2, Norma Rubini³, João Paulo Reis Neto ${ }^{4}$, Juliana Busch²,4 Júlio Ferro5, Márcio Vinicius Balzan6, Zuleid Dantas Linhares Matar7, Guilherme Crespo ${ }^{8}$, Wilson Follador²,

DOI: $10.21115 / \mathrm{JBES} . v 13 . n 2 . p 221-8$

\section{Palavras-chave:}

asma, sustentabilidade, gestor, contrapartida, risk-sharing

\section{Keywords:}

asthma, sustainability, manager, counterpart, risk-sharing

\section{RESUMO}

Realizou-se, no dia 29 de agosto de 2020, um encontro virtual com gestores, representantes de associações de pacientes e médicos prescritores envolvidos no tratamento da asma, com o objetivo de discutir a necessidade e a viabilidade da incorporação de novas tecnologias para o tratamento dessa patologia. A asma é uma enfermidade caracterizada pela inflamação crônica das vias aéreas. É a principal causa de absenteísmo escolar e laboral e estima-se que seja responsável por até cinco mortes diárias em nosso país. Em pesquisa efetuada em operadora de autogestão, com vidas espaIhadas por todo o país, observou-se que essa patologia (associada à doença pulmonar obstrutiva crônica - DPOC) acomete quase 7\% dos seus segurados e que a utilização do plano foi, nesse grupo, 1,9x maior para consultas, 1,6x maior para exames, 2,5x maior para terapias e 2,9x maior para internações, resultando em um aumento de $25,5 \%$ nos gastos assistenciais. Observou-se que é de elevada importância que o gestor tenha um profundo conhecimento de sua carteira e que priorize toda a linha de cuidado do paciente. Dessa maneira, atuando diretamente no controle da severidade da patologia, terão os melhores resultados de qualidade de vida e restringirão os doentes que necessitarão de medicações mais modernas e, também, mais caras, com resultado óbvio no controle de custos. A asma ainda não tem, em geral, para os gestores de operadoras de saúde privadas, no Brasil, uma importância tão grande na sinistralidade das suas carteiras. Os novos imunobiológicos são úteis e efetivos e alguma contrapartida por parte do fabricante, como, por exemplo, o compartilhamento de riscos, pode ser necessária para uma incorporação desse arsenal no Rol de produtos que serão disponibilizados para os pacientes que deles necessitarem.

Recebido em: 05/04/2021. Aprovado para publicação em: 07/05/2021

1. Professor de Bioética, Cátedra Unesco de Bioética, Universidade de Brasília, Brasília, DF, Brasil.

2. Sociedade Internacional de Farmacoeconomia e Pesquisa de Desfechos (ISPOR), Capítulo Brasil, Goiânia, GO, Brasil.

3. Professora titular de Alergia e Imunologia, Universidade Federal do Estado do Rio de Janeiro, Rio de Janeiro, RJ, Brasil.

4. Caixa de Previdência e Assistência dos Servidores da Fundação Nacional de Saúde (Capesesp), Rio de Janeiro, RJ, Brasil.

5. Hospital das Clínicas, Universidade Federal de Goiânia, Goiânia, GO, Brasil.

6. Regulação de Sinistro, Medicina de Grupo e Unimed do interior de São Paulo, Jundiaí, SP, Brasil.

7. Presidente da Associação Brasileira de Asmáticos (ABRA), São Paulo, SP, Brasil.

8. Diretor Médico, Athena, São Paulo, SP, Brasil.

9. Sano-Efiko, São Paulo, SP, Brasil.

Autor correspondente: Marcos Santos. Rua Camilo Flamarion, quadra 10, lote 1, Serrinha, Goiânia, GO, Brasil. CEP: 74835-010.

E-mail: marcosrxt@gmail.com 
control of the severity of the pathology, they will have the best quality of life results and will restrict the number of patients who will need more modern and also more expensive medications, with obvious impact on costs. Asthma still does not have, for the manager of a Private Health Operator, in Brazil, such importance in the expenses of his portfolio. The new immunobiologicals are useful, effective and some counterpart on the part of the manufacturer, such as, for example, the risk-share agreements, may be necessary for the incorporation of this into the arsenal of products that will be made available to patients that need them.

\section{Introdução}

A asma é uma patologia caracterizada pela inflamação crônica da via aérea associada a uma limitação variável do fluxo de ar (Rehman et al., 2018). Causa enorme desconforto ao paciente e pode, nos casos mais graves, levar à morte se os cuidados adequados não forem implementados. Estima-se que, no Brasil, a asma atinja quase 9 milhões de pessoas, o que equivale a pouco mais de $4 \%$ da população. A asma é a principal causa de absenteísmo escolar e laboral em nosso país. As formas mais graves correspondem a 5\% a 10\% dos casos. Estima-se que haja até cinco mortes diárias por causa dessa patologia no Brasil (GINA, 2019).

Em nosso país, a asma também é responsável por um número representativo de internações hospitalares. Ocorriam, por aqui, aproximadamente, 370 mil internações por asma, anualmente, em 2010, constituindo essa a quarta principal causa de hospitalizações pelo Sistema Único de Saúde - SUS (Rodrigues et al., 2010), embora uma tendência recente de diminuição desses números tenha sido observada nos últimos anos, com a universalização do uso dos corticoides inalatórios (Costa et al., 2018).

A asma grave contribui, segundo estimativas, com 50\% dos custos do tratamento dessa patologia. No Canadá, essa fração supera os 60\% (Sadatsafavi et al., 2010). É importante diferenciar, no entanto, asma grave de asma não controlada. A asma grave requer tratamento baseado na etapa 5 da GINA (Global Initiative for Asthma): corticoides inalatórios orais em dose alta associados a beta-agonistas de longa duração ou antagonistas dos receptores de leucotrienos/teofilina no último ano ou, como terceira opção, uso de corticoide oral em mais de metade do último ano para prevenção de crises. Também é definida como asma grave aquela que permanece sem controle, a despeito da terapia adequadamente implementada. Já, por outro lado, asma não controlada é definida como aquela com controle pobre dos sintomas, com exacerbações graves e frequentes (dois ou mais usos de corticoides orais por mais de três dias) no último ano, pelo menos uma hospitalização com internação em unidade de terapia intensiva (UTI) ou ventilação mecânica no último ano ou limitação do fluxo aéreo [volume expiratório forçado no primeiro segundo (VEF1) < 90\% do predito após broncodilatação na presença de relação VEF1/capacidade vital forçada (CFV) reduzida] (Chung et al., 2014). O paciente com asma grave tem cinco vezes mais chances de sofrer uma exacerbação da sua patologia, 15 vezes mais chances de necessitar de uma consulta médica de emergência, um risco 20 vezes maior de hospitalização e uma chance seis vezes maior de uma exacerbação grave após uma exacerbação grave nos últimos três meses (Kupczyk et al., 2014; Miller et al., 2007).

Mais especificamente, sobre a asma grave no Brasil, 90\% dos pacientes foram hospitalizados pelo menos uma vez em virtude de sua patologia. Aproximadamente 2/3 deles relatam cinco ou mais episódios de internação, tendo a metade sido hospitalizada pelo menos uma vez durante o ano anterior a esse levantamento, publicado por Carvalho-Pinto et al. Metade dos pacientes com asma grave foi internada em UTI e 38\% deles necessitaram de intubação orotraqueal (de Carvalho-Pinto et al., 2012).

A terapia biológica é uma estratégia inovadora que, recentemente, tem sido incorporada ao arsenal terapêutico do médico responsável pelo tratamento da asma. Os biológicos são uma classe diversa e heterogênea de produtos. Existem hormônios, fatores de crescimento e diferenciação celular, enzimas, anticoagulantes e os modernos anticorpos monoclonais. Especificamente, os imunobiológicos são moléculas de alto peso molecular que atuam no sistema imune, compreendendo três classes principais: anticorpos monoclonais, proteínas de fusão e citocinas humanas. No caso da asma, só estão disponíveis, comercialmente, anticorpos monoclonais (Tabela 1).

Com o objetivo de discutir o impacto da asma na sinistralidade das operadoras de saúde e a melhor maneira de avaliar e proceder à incorporação desses novos medicamentos que vêm enriquecer o arsenal terapêutico do médico encarregado do tratamento dessa patologia, reunimos, de maneira virtual, no último 29 de agosto, vários especialistas no tema, cobrindo todos os stakeholders envolvidos - médicos prescritores, gestores de operadoras de autogestão, medicina de grupo e cooperativa, além de especialistas em economia da saúde -, para propor alternativas e viabilizar o acesso dos pacientes ao melhor tratamento que lhes pode ser disponibilizado.

\section{As operadoras de saúde}

Após a abertura feita pelo deputado federal Pedro Westphalen, que descreveu sua intensa atividade parlamentar objetivando melhorar o sistema de saúde público brasileiro (o deputado é presidente da Comissão Parlamentar de Doenças Pulmonares) e, depois da introdução a respeito da patologia feita pela Dra. Norma Rubini, cujas informações principais fo- 
ram descritas na introdução deste artigo, passou-se a palavra aos gestores de operadoras de saúde, com a demanda de descreverem qual era o tamanho do problema "asma" nas suas respetivas operadoras. Iniciou a apresentação o Dr. João Paulo, presidente da Capesesp. Ele ressaltou que as operadoras de saúde vêm enfrentando um cenário bastante complicado de envelhecimento da população, o que leva, inexoravelmente, a um aumento do custo dos cuidados. Dada a situação econômica complicada em que vivemos, atualmente, é sensível a diminuição da população jovem entre os segurados.

Em pesquisa que buscava identificar o perfil epidemiológico da população atendida pela Capesesp, foram realizadas entrevistas por correio ou telefone. E o resultado mostrou que $6,7 \%$ dos segurados referiam sofrer de asma ou doença pulmonar obstrutiva crônica (DPOC). Aproximadamente 1/5 dessa população tinha o diagnóstico há mais de cinco anos e metade fazia uso de alguma medicação de maneira crônica. Em torno de $1 / 4$ dos indivíduos tinham se afastado do trabalho, e em $12,5 \%$ dos casos esse afastamento tinha sido superior a 15 dias.

Sabemos que existem fortes evidências de que a saúde percebida pelo individuo é um excelente preditor da sua saúde objetiva e que indivíduos que consideram a sua saúde como sendo ruim apresentam um risco aumentado de hospitalização, em comparação àqueles que julgam a sua saúde como excelente (Nielsen et al., 2014; Pagotto et al., 2011). Em torno de $7 \%$ da população com DPOC/asma avaliou a sua saúde como muito ruim e 33\%, como regular. A avaliação de 60\% era de que sua saúde era boa ou muito boa. Em seguida, avaliou-se a taxa de utilização do plano entre os segurados que tinham referido asma ou DPOC como diagnóstico. E isso foi comparado com quem não referia ser portador dessas patologias. As taxas, em ambos os grupos, foram multiplicadas pelo custo unitário dos eventos para que fosse estimado o custo per capita anual. Esses resultados foram extrapolados para o total de vidas, conforme a prevalência dos problemas de saúde encontrados. A amostra diagnosticada com asma incluiu 50 homens com idade média de 48,8 anos e 81 muIheres, estas com idade média de 56 anos. A utilização do

Tabela 1. Principais anticorpos monoclonais (em ordem alfabética) registrados no Brasil para tratamento da asma grave

\begin{tabular}{llll}
\hline Droga & $\begin{array}{l}\text { Nome } \\
\text { comercial }\end{array}$ & $\begin{array}{l}\text { Empresa } \\
\text { fabricante }\end{array}$ & Indicação \\
\hline Benralizumabe & Fasenra & AstraZeneca & $\begin{array}{l}\text { Asma grave } \\
\text { eosinofílica }\end{array}$ \\
\hline Dupilumabe & Dupixent & Sanofi & $\begin{array}{l}\text { Asma grave } \\
\text { inflamação II }\end{array}$ \\
\hline Mepolizumabe & Nucala & GSK & $\begin{array}{l}\text { Asma grave } \\
\text { eosinofílica }\end{array}$ \\
\hline Omalizumabe & Xolair & Novartis & $\begin{array}{l}\text { Asma grave } \\
\text { alérgica }\end{array}$ \\
\hline
\end{tabular}

plano foi, nesse grupo, 1,9x maior para consultas, 1,6x maior para exames, 2,5x maior para terapias e 2,9x maior para internações. O custo anual dos pacientes com asma foi de $\mathrm{R} \$ 21.494 .186,00$ versus $\mathrm{R} \$ 17.123 .138,00$ para os pacientes sem asma. Considerando a prevalência estimada e o custo incremental, o impacto anual da asma foi de R\$ 4.371.048,00. Com isso, os autores concluíram que, apesar da queda da morbidade hospitalar e da mortalidade por asma observada na última década, encontrou-se, no presente estudo, uma maior utilização do plano de saúde pelo grupo composto pelos pacientes asmáticos, resultando em um aumento de 25,5\% nos gastos assistenciais. Desse modo, os gestores devem estar atentos a essa doença, como forma de promover o uso racional e eficiente dos recursos, a fim de possibilitar o acesso às novas e promissoras tecnologias que chegam ao mercado, que, apesar do comprovado benefício para o paciente, têm em comum o custo elevado.

Em seguida, os Drs. Julio Ferro e Marcio Vinícius Balzan reforçaram o que havia sido dito anteriormente, compartilhando das preocupações e conclusões para a medicina de grupo e para cooperativas. É de elevadíssima importância o conhecimento que o gestor deve ter de sua carteira. Acrescentaram que centros especializados podem ser úteis na otimização da utilização de tecnologias inovadoras, evitando o desperdício e a utilização sem critérios de eficácia comprovada, e que as melhores decisões são aquelas que, de posse dessa carga de informação, levam em consideração, além das evidências clínicas, conhecimentos médicos e econômicos. Ainda, infelizmente, valoriza-se muito pouco a prevenção como forma de contenção de custos.

A Dra. Zuleid reforçou, em seguida, que, a despeito de todas as limitações acima descritas, não se pode perder de vista que o paciente tem sempre que receber o melhor tratamento que Ihe é disponível. E o protocolo do SUS, por exemplo, não é atualizado desde o ano de 2013. Desde então, muita coisa mudou no tratamento dessa patologia: as crianças beneficiam-se do uso do corticoide inalatório, prevenindo-se o remodelamento pulmonar; não se utiliza mais broncodilatador de ação rápida isoladamente em nenhuma etapa do tratamento; a GINA, citada anteriormente neste artigo, incorporou broncodilatadores antimuscarínicos de longa ação a partir dos 6 anos, na etapa 4 (GINA); o conhecimento dos fenótipos direciona para uma terapia sob medida; e, finalmente, houve o aparecimento dos imunobiológicos. A mesma doutora defendeu, quanto aos medicamentos, que não basta o princípio ativo: é importante a apresentação. Sprays são mais democráticos. Espaçadores são fundamentais para a boa deposição. A tecnologia de micropartículas permite atingir as pequenas vias aéreas, protegendo do remodelamento pulmonar. É importante lembrar que há a possibilidade de se utilizar o mesmo medicamento para controle dos sintomas e, também, na exacerbação deles, para resgate. $E$, finalmente, nunca é demais lembrar que pacientes são di- 
ferentes e, portanto, necessitam de tratamentos diferentes, segundo as suas necessidades e condições pessoais.

A Dra. Zuleid finalizou sua fala citando o estudo publicado por Comaru et al., que descreve que, desde 2011, o SUS começou a distribuir gratuitamente beclometasona e salbutamol a pacientes com asma. Os autores observaram uma importante diminuição nas taxas de internação hospitalar após a implementação dessa política pública, sugerindo que a distribuição gratuita de remédios de custo relativamente baixo tem importância preponderante como estratégia de melhora dos resultados e de controle de custo dessa patologia (Comaru et al., 2016).

\section{Estratégias alternativas de incorporação tecnológica}

O Dr. Guilherme Crespo, o Dr. Wilson Follador e o Dr. Marcos Santos foram os responsáveis pela fase final do evento, que objetivava discutir, diante da importância crescente que a asma vem atingindo na sinistralidade das operadoras de saúde, conforme claramente dimensionado anteriormente e, diante da apresentação de novas terapias imunobiológicas para o controle dos casos graves dessa patologia, estratégias alternativas objetivando à incorporação.

O Dr. Marcos falou sobre a ética no tratamento da asma. Ética é o estudo da moral. E moral, palavra de origem grega, é o costume, hábito de uma sociedade. Ética é a ciência dos costumes. Objetiva determinar o que é certo. $O$ método desenvolvido pela nossa sociedade ocidental, para a incorporação tecnológica, foi a farmaeconomia, baseada numa ética consequencialista, a do utilitarismo. O objetivo é conseguir os melhores resultados, e por melhores entenda-se o maior bem-estar para o maior número de pessoas pelo maior tempo possível (Santos, 2014).

Podemos dividir a história da ATS, a avaliação de tecnologia de saúde, em três fases (Tabela 2). A primeira foi aquela vivida na segunda metade do século passado, quando surgiu essa ciência. No pós-guerra, definiu-se saúde como um completo bem-estar físico, mental e social. Nessa toada, o movimento sanitário brasileiro cravou na Constituição: saúde é direito de todos e dever do Estado, garantido mediante políticas sociais e econômicas que visem à redução do risco de doença e de outros agravos e ao acesso universal e igualitário às ações e serviços para sua promoção, proteção e recuperação. Nessa primeira fase, podia-se ser utópico. Os custos da saúde podiam ser considerados, em comparação com o que vivenciamos hoje, como baixos. Era mais fácil, do ponto de vista ético, aceitar a divisão.

Posteriormente, inicia-se a fase que vivenciamos hoje, intermediária, quando se vislumbra que as curvas de recursos disponíveis e custos vão, em algum momento, se cruzar. Ainda há um largo trabalho de gestão a ser feito para que esse cruzamento se dê em um futuro o mais distante possível. Aqui, a ATS tem sua real utilidade. Inicialmente, elimina-se o desperdício. Paralelamente, restringe-se, subindo o limiar de eficácia exigido da evidência, o acesso a tratamentos menos efetivos. Mas não há, até onde a vista alcança, sinal de que o crescimento dos custos tenha atingido algum tipo de limiar. Na ausência dele, por mais gestão efetiva que se pratique, uma hora esse cruzamento vai ocorrer. A sociedade está sendo chamada a decidir quanto aceita gastar com saúde. Dez por cento do que produz? Vinte? Trinta? Qual é o limite? Esse cruzamento vai em algum momento ocorrer. Teremos, então, a terceira fase, aquela em que tratamentos, ainda que efetivos, ainda que custo-efetivos, não serão incorporados. Simplesmente porque não haverá recursos para tanto. Nem nos países mais ricos, que serão os últimos, muito provavelmente, a atingir essa fase, que chamaremos de pós-ATS, em que as escolhas vão ficar muito mais complicadas do que são hoje. Os nossos critérios, aceitos hoje, vão se tornar insuficientes.

Mas ainda estamos na segunda fase. Aqui, o trabalho do gestor objetivando à otimização ainda é factível. E precisamos pensar em alternativas. $O$ erro médico ainda é a terceira causa

Tabela 2. Fases da ATS

\begin{tabular}{|c|c|c|c|}
\hline Fase & Início & Fim & Características \\
\hline 1 & $\begin{array}{l}\text { Pós-guerra: } \\
\text { criação da } \\
\text { OMS }\end{array}$ & $\begin{array}{l}\text { Final da década de 1980, } \\
\text { início da década de } \\
1990\end{array}$ & $\begin{array}{l}\text { - Tecnologias relativamente baratas } \\
\text { - Visão utópica da saúde (vide OMS, Welfare State europeu e, no Brasil, movimento } \\
\text { sanitário brasileiro) } \\
\text { - Busca da saúde universal, estabelecimento de metas mundiais (Alma-Ata) }\end{array}$ \\
\hline 2 & $\begin{array}{l}\text { Década de } \\
1990\end{array}$ & $?$ & $\begin{array}{l}\text { - Tecnologias com custo exponencialmente crescente } \\
\text { - Metas não foram atingidas em nível global } \\
\text { - Gerenciamento com capacidade de melhoria no acesso } \\
\text { - Aumento significativo da expectativa de vida, principalmente da camada mais } \\
\text { favorecida da população } \\
\text { - Estratégias (com graus variados de efetividade) de controle (temporário) dos custos }\end{array}$ \\
\hline 3 & $?$ & Ruptura? & $\begin{array}{l}\text { - Manutenção do crescimento exponencial dos custos } \\
\text { - Aumento relativo significativo dos recursos investidos em saúde até o limite que a } \\
\text { - Gociedade está disposta a dispender } \\
\text { - Aumenciamento, ainda que efetivo, não é suficiente para melhoria do acesso } \\
\text { - Augualdade dos resultados obtidos em nível global }\end{array}$ \\
\hline
\end{tabular}


de morte nos hospitais dos Estados Unidos (Makary \& Daniel, 2016). E há, também, o desperdício. Segundo Quirós \& Puerta, a mercantilização da medicina converteu o enfermo em um consumidor como outro qualquer, cujo direito de requerer, segundo seu próprio desejo (e de escolher diante de possibilidades que ele mesmo julga disponíveis), deve, obviamente, ser limitado (e pactuado democraticamente), ainda que qualquer política que trabalhe nessa direção se converta, instantaneamente, em bastante antipática (Quirós \& Puerta, 2009).

Em primeiro lugar, como bem pontuou o Dr. Guilherme Crespo, é preciso ficar claro que os tratamentos mais caros são úteis, conforme aponta a evidência, para os pacientes mais graves, e não para aqueles pacientes não controlados que não receberam o tratamento mais simples e mais barato que Ihes seria, se bem aplicado, efetivo. Essa primeira seleção, que é efetivada quando o gestor olha atentamente para toda a linha de cuidado do paciente, é extremamente efetiva. Diminuem-se, sem prejudicar o estado de saúde dos doentes, aqueles que realmente vão precisar dos remédios mais caros. E a experiência mostra que, por mais óbvia que essa afirmação possa ser, essa estratégia ainda traz resultados sensíveis em muitas operadoras espalhadas pelo país, porque, ainda que intuitiva, lógica, não tem aplicação fácil. Demanda, entre outras coisas, um intenso programa de educação do segurado. A medicina preventiva atinge aquele indivíduo que ou não está doente ou, se está, ainda não tem uma patologia que atrapalhe sobremaneira a sua vida. Especificamente no caso da asma, que nos interessa na discussão atual, buscar melhora da qualidade vida do paciente com asma significa atuar diretamente na severidade da doença. A severidade da patologia é o fator que mais influi na qualidade de vida desses pacientes (Hossny et al., 2017).

O próximo passo é ter centros especializados, com profissionais experientes, largamente treinados para o acompanhamento dos casos mais complexos. Os centros de referência vão ter - a literatura mostra - muito mais sucesso no uso de tratamentos mais complexos. O que, ao final, também gera otimização dos recursos. Mas o que fazer quando ainda não temos resultados definitivos da evidência científica? Ou quando a evidência é robusta, mas há dúvidas a respeito do subgrupo de indivíduos que melhor se adaptarão aos tratamentos? Aqui, há incertezas que demandam tempo para o seu esclarecimento. Mas esperar esse tempo pode ser uma oportunidade perdida para um sem número de doentes. Quem fica com o risco?

Uma alternativa foi a estratégia detalhadamente explicada pelo Dr. Wilson. Por que não um compartilhamento dos riscos entre operadora de saúde e indústria farmacêutica? Todos os envolvidos no processo de incorporação tecnológica estão expostos a riscos. A indústria pode ter dificuldade em vender o seu produto, o financiador pode pagar por uma tecnologia que, no futuro, não se mostre efetiva e, finalmente, o paciente pode receber um tratamento que não seja eficiente ou que ele, deste, não necessite. Risk-sharing, ou compartilhamento de riscos, é um modelo de negócios no qual o fornecedor da tecnologia e o pagador concordam em estabelecer, entre si, um acordo em que ambas as partes assumem riscos de perdas e ganhos. No modelo mais geral, o provedor de serviços aplica a tecnologia. Há uma monitorização por um terceiro elemento e, no caso de funcionamento, com desfechos relevantes previamente definidos, há a remuneração. Na ausência da obtenção do desfecho, não há o pagamento.

Esse acordo pode trazer benefícios: aos pacientes, como acesso a tratamentos restritos; aos provedores de serviços de saúde, como redução dos atritos com as operadoras e ampliação do arsenal terapêutico; aos gestores do sistema de saúde, porque conseguem um melhor controle do impacto orçamentário ocasionado pelas novas tecnologias, com redução das incertezas inerentes ao processo de incorporação; e, finalmente, à indústria farmacêutica, que tem um acesso mais rápido e efetivo ao mercado, com redução dos riscos relacionados ao uso inapropriado da sua tecnologia. Há, também, é preciso ressaltar, algumas desvantagens, como em todo acordo. Os pacientes estão sujeitos ao risco de interrupção do fornecimento caso o contrato seja encerrado. Os provedores podem ser forçados a atuar em novas atividades, como monitorização e controle (o que, a longo prazo, tende a deixar de ser uma desvantagem); gestores de saúde enfrentam, frequentemente, as dificuldades inerentes na definição dos indicadores mais pertinentes; e, por fim, a indústria enfrentará, em troca da rapidez no acesso e no uso da tecnologia, uma potencial redução nos ganhos, além da necessidade do custeio de processos acessórios àquele do compartilhamento dos riscos.

Para todos os envolvidos, há sempre os obstáculos legais, os riscos de infidelidade dos partícipes, a possibilidade de haver baixa adesão aos protocolos de tratamento e a elevada dependência da competência daquele que ficar responsável pela definição da obtenção ou não do desfecho combinado. É preciso que haja adaptações às necessidades específicas de cada operadora e de cada tecnologia. O Dr. Wilson concluiu a sua apresentação dizendo que não há uma receita pronta, um modelo de remuneração ideal, mas que distribuir os ganhos pode ajudar. Pode ser uma forma de equilibrar as forças e tornar o sistema mais sustentável. Há vários modelos de remuneração fartamente descritos na literatura e o risk-sharing é um grande passo na direção de modelos assistenciais baseados em valor.

\section{Pesquisa pós-evento}

Após o evento, os participantes foram convidados a responder a algumas perguntas. A seguir, descrevemos os resultados. Inicialmente perguntamos aos gestores qual era a importância da asma para as suas operadoras, no que se referia a custos, número de pacientes e sinistralidade. A maioria dos respondedores atestou que a tal importância era, ainda, pequena (Figura 1). 
Em seguida, perguntamos aos gestores qual era a sua opinião a respeito dos imunobiológicos no tratamento da asma. Seriam esses medicamentos eficazes? A maioria (80\%) respondeu que sim, enquanto $20 \%$ entendem que esses medicamentos são "extremamente eficazes" (Figura 2).

Mas, apesar disso, a maioria também entende que esses medicamentos são caros (Figura 3).
Finalmente, perguntamos aos gestores se, na ocorrência da não incorporação dos imunobiológicos no Rol de procedimentos de cobertura obrigatória da Agência Nacional de Saúde, qual seria a probabilidade de que ela/ele advogasse pela incorporação deles no protocolo de sua operadora. A maioria respondeu que tal atitude dependeria de uma contrapartida (Figura 4).

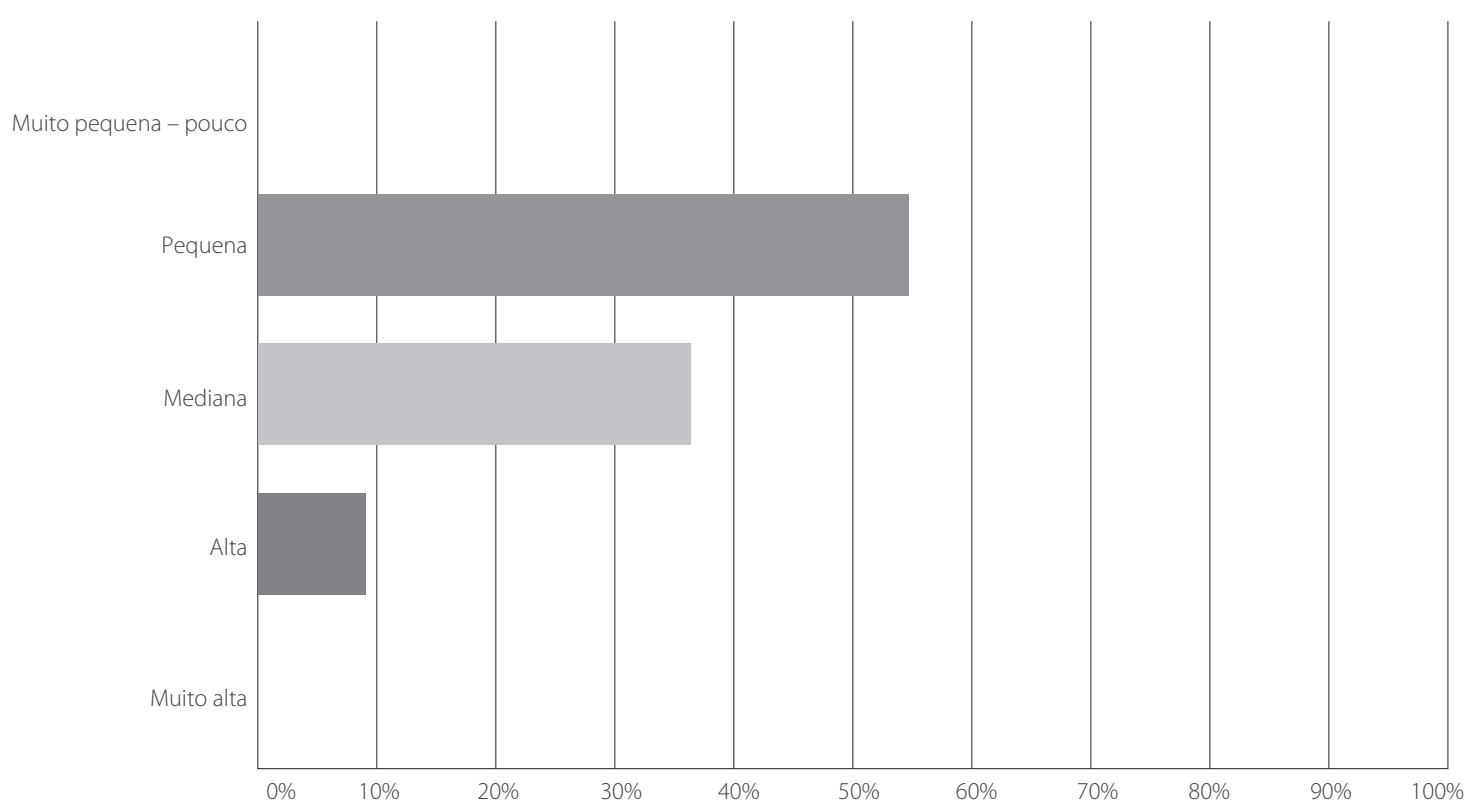

Figura 1. Importância da asma para a sua operadora.

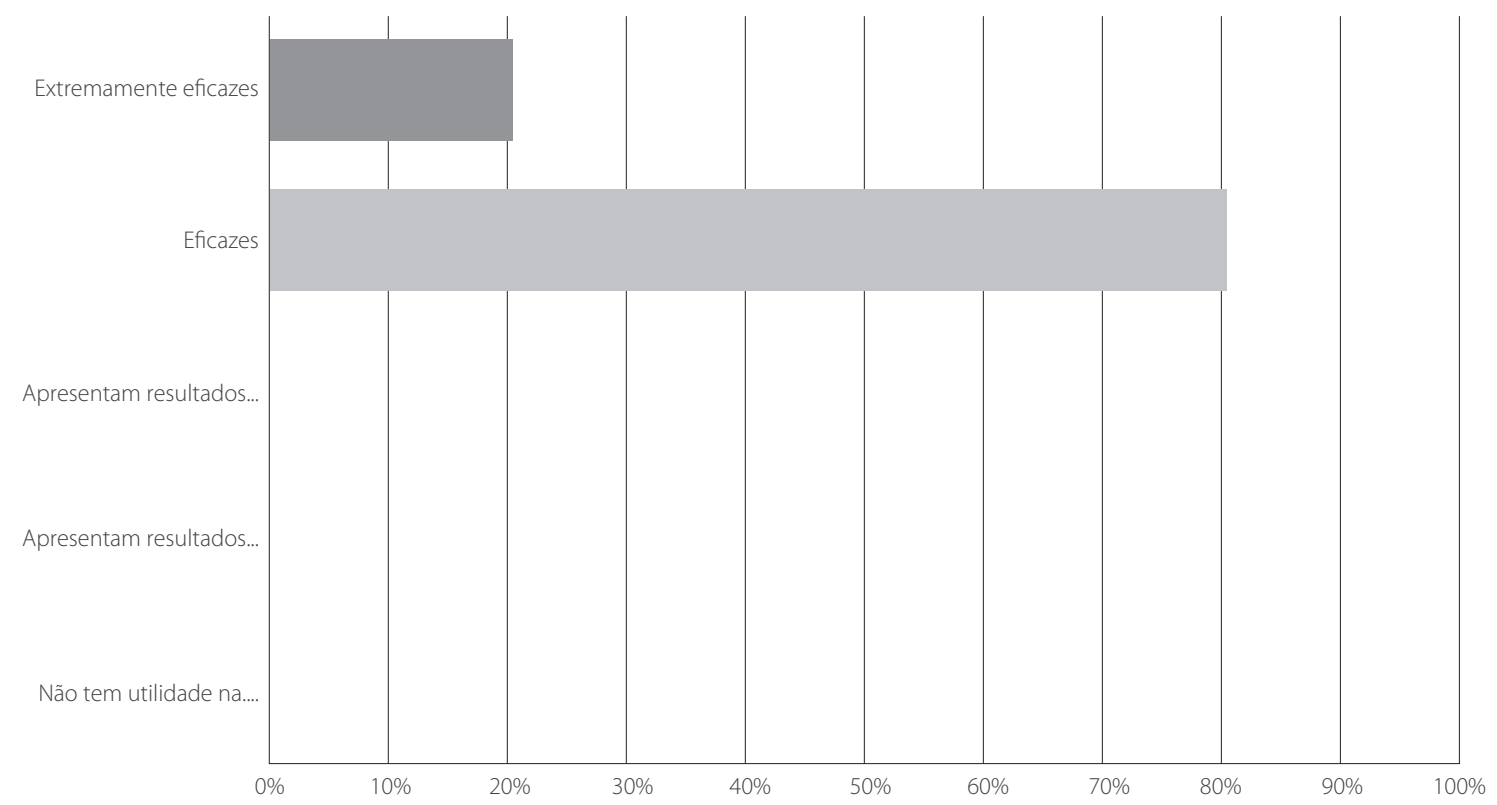

Figura 2. Eficácia dos imunobiológicos no tratamento da asma. 


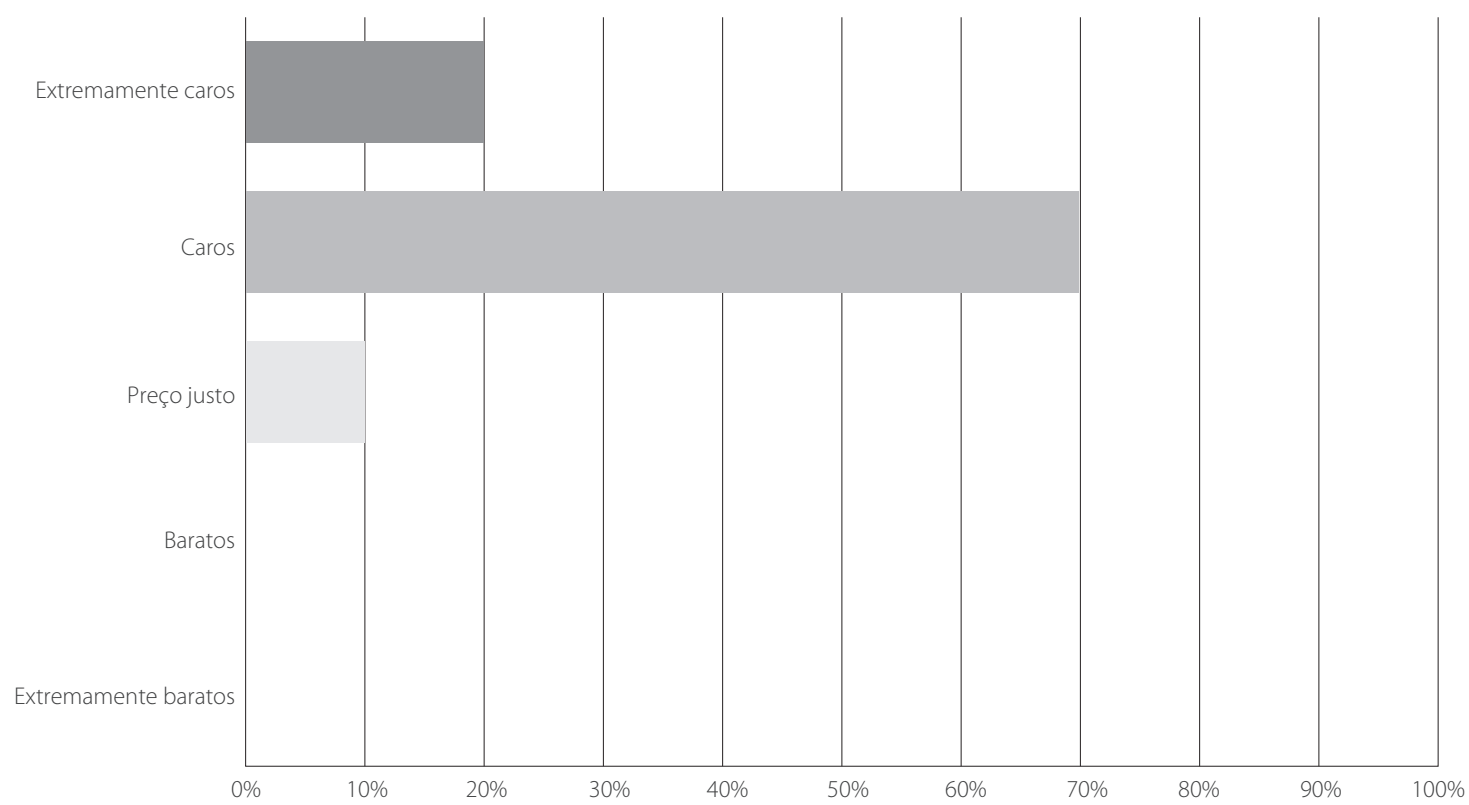

Figura 3. Valoração sobre o preço dos imunobiológicos no tratamento da asma.

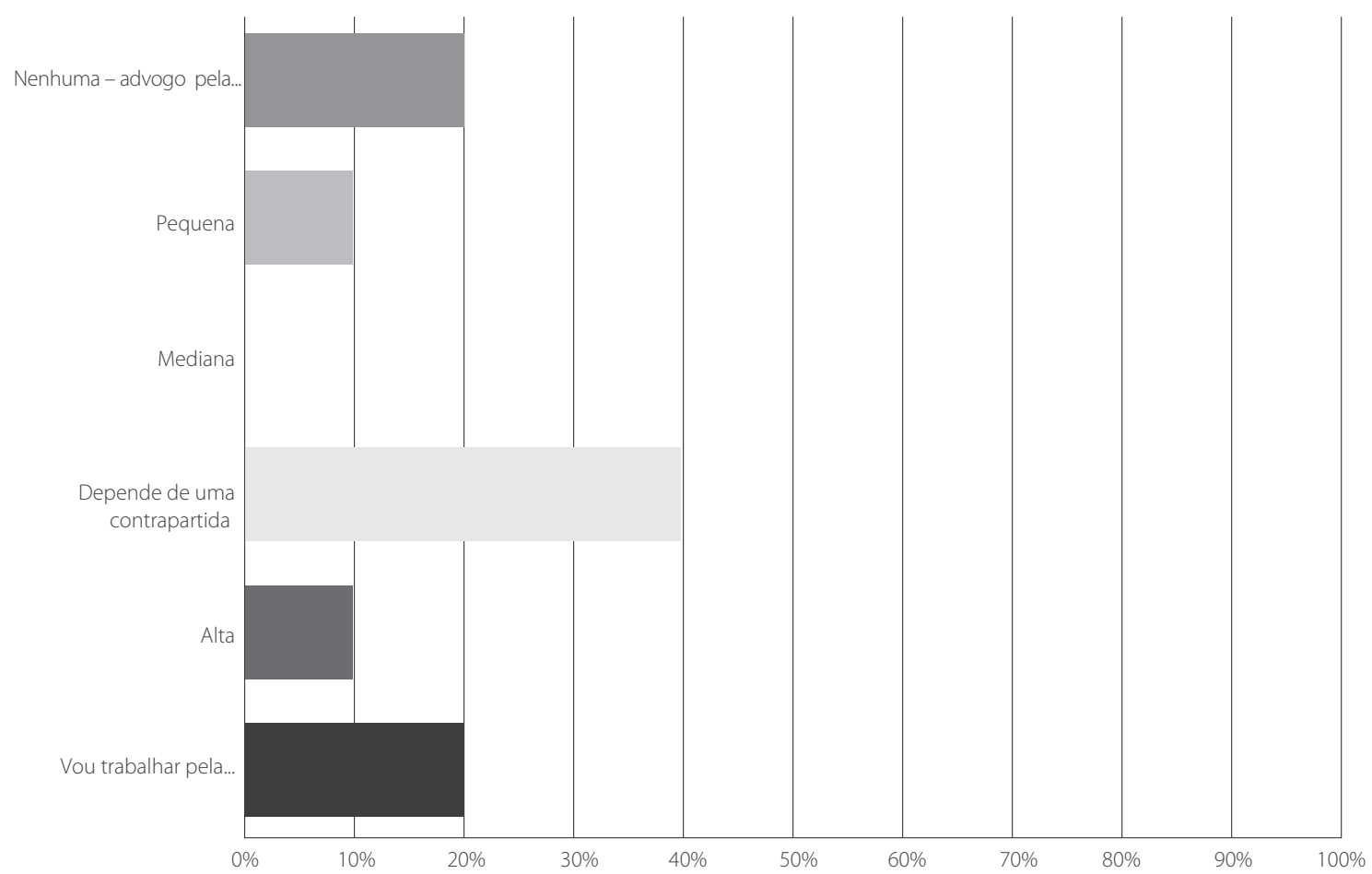

Figura 4. Probabilidade de que o gestor defenda a incorporação dos imunobiológicos para o tratamento da asma no protocolo de sua operadora, caso não haja incorporação no Rol da Agência Nacional de Saúde.

\section{Conclusões}

Observamos, a partir do exposto, que, embora a asma ainda não seja responsável por uma parcela preocupante dos gastos e da sinistralidade das operadoras de saúde privadas no Brasil, esse cenário é dinâmico e pode vir a ser alterado no futuro. Novas tecnologias estão sendo apresentadas e uma fotografia atual indica que muitas vidas ainda se perdem e mui- tas internações (muitas delas em UTI) ainda são necessárias, não porque os pacientes tenham asma grave, mas porque têm asma não controlada. Com uma disseminação do acesso, com consequente aumento do uso de medicações inovadoras, é possível que esse cenário melhore, mas vai trazer consigo um inevitável aumento dos custos. Contrapartidas são necessárias (e neste artigo algumas delas são descritas) para que nossos pacientes tenham um melhor controle de 
suas patologias. É isso, conforme mostra a literatura, que mais vai interferir nas suas respectivas qualidades de vida.

Ações proativas são necessárias. Não fazer nada, neste momento, pode significar não atentar para uma necessidade não atendida e, também, a manutenção de altos custos, secundários a internações, muitas delas em terapia intensiva. Esse encontro mostrou que esse gasto não é percebido como muito alto neste momento, mas, seguramente, não é o mais efetivo para um número considerável de pacientes.

\section{Agradecimentos}

Agradecemos imensamente a Henrique Perdigão Oliveira e Ribeiro, pelo competente trabalho de divulgação do evento citado neste paper e por sua enorme e insubstituível contribuição para o sucesso dele.

\section{Referências bibliográficas}

Chung KF, Wenzel SE, Brozek JL, Bush A, Castro M, Sterk PJ, et al. International ERS/ATS guidelines on definition, evaluation and treatment of severe asthma. Eur Respir J. 2014;43(2):343-73.

Comaru T, Pitrez PM, Friedrich FO, Silveira VD, Pinto LA. Free asthma medications reduces hospital admissions in Brazil (Free asthma drugs reduces hospitalizations in Brazil). Respir Med. 2016;121:21-5.

Costa E, Caetano R, Werneck GL, Bregman M, Araújo DV, Rufino R. Estimated cost of asthma in outpatient treatment: a real-world study. Rev Saúde Pública. 2018;52.

de Carvalho-Pinto RM, Cukier A, Angelini L, Antonangelo L, Mauad T, Dolhnikoff M, et al. Clinical characteristics and possible phenotypes of an adult severe asthma population. Respir Med. 2012;106(1):47-56.
GINA - Global Initiative for Asthma. GINA Report, Global Strategy for Asthma Management and Prevention. 2019. Available from: https://ginasthma. org/gina-reports/

Hossny E, Caraballo L, Casale T, El-Gamal Y, Rosenwasser L. Severe asthma and quality of life. World Allergy Organ J. 2017;10(1):28.

Kupczyk M, ten Brinke A, Sterk PJ, Bel EH, Papi A, Chanez P, et al. Frequent exacerbators - a distinct phenotype of severe asthma. Clin Exp Allergy. 2014;44(2):212-21.

Makary MA, Daniel M. Medical error - the third leading cause of death in the US. BMJ. 2016;353:i2139.

Miller MK, Lee JH, Miller DP, Wenzel SE; TENOR Study Group. Recent asthma exacerbations: a key predictor of future exacerbations. Respir Med. 2007;101(3):481-9.

Nielsen AB, Siersma V, Waldemar G, Waldorff FB. The predictive value of self-rated health in the presence of subjective memory complaints on permanent nursing home placement in elderly primary care patients over 4-year follow-up. Age Ageing. 2014;43(1):50-7.

Pagotto V, Nakatani AY, Silveira EA. [Factors associated with poor self-rated health in elderly users of the Brazilian Unified National Health System]. Cad Saude Publica. 2011;27(8):1593-602.

Quirós J, Puerta J. Tecnología, demanda social y medicina del deseo. Med Clin (Barc). 2009;133:671-5.

Rehman A, Amin F, Sadeeqa S. Prevalence of asthma and its management: A review. J Pak Med Assoc. 2018;68(12):1823-7.

Rodrigues PCO, Ignotti E, Rosa AM, Hacon SS. Spatial distribution of asthmarelated hospitalizations of the elderly in the Brazilian Amazon. Rev Bras Epidemiol. 2010;13(3):523-32.

Sadatsafavi M, Lynd L, Marra C, Carleton B, Tan WC, Sullivan S, et al. Direct health care costs associated with asthma in British Columbia. Can Respir J. 2010;17(2):74-80.

Santos M. Princípios básicos de bioética aplicados em economia da saúde e em auditoria médica. JBES. 2014;6(2):97-101. 